\title{
On the Privacy Practices of Just Plain Sites
}

\author{
Amirhossein Aleyasen \\ University of Illinois \\ Urbana, IL, USA \\ aleyase2@illinois.edu
}

Oleksii Starov

Stony Brook University

Stony Brook, NY, USA

Oleksii.Starov@stonybrook.edu Pittsburgh, PA, USA

APA25@pitt.edu

\author{
Jeff Shrager \\ Corresponding author \\ Commerce Net \\ Palo Alto, CA, USA \\ jshrager@stanford.edu
}

\author{
Allan Schiffman \\ Commerce Net \\ Palo Alto, CA, USA \\ ams@commerce.net
}

\begin{abstract}
In addition to visiting popular sites such as Facebook and Google, web users often visit more modest sites, such as those operated by bloggers, or by local organizations such as schools. Such sites, which we call "Just Plain Sites" (JPSs), are likely to inadvertently present greater privacy risks than highly popular sites, because they are unable to afford privacy expertise. To assess the prevalence of the privacy risks to which JPSs may inadvertently be exposing their visitors, we examined privacy practices that could be observed by analysis of JPS landing pages. We found that many JPSs collect a great deal of information from their visitors, and share a great deal of information about their visitors with third parties. For example, we found that an average of 7 third party organizations are informed when a user visits a JPS. Many JPSs additionally permit a great deal of tracking of their visitors. For example, we found that third party cookies are used by more than $50 \%$ of JPSs. We also found that many JPSs use deprecated or unsafe security practices. Our goal is not to scold JPS operators, but to raise awareness of these facts among both JPS operators and visitors, possibly encouraging operators to take greater care in their implementations, and visitors to take greater care in how, when, and what they share.
\end{abstract}

\section{Categories and Subject Descriptors}

K.4.1 [Computers and Society]: Public Policy IssuesPrivacy; K.6.5 [Management of Computing and Information Systems]: Security and Protection-Unauthorized access

\section{Keywords}

Web privacy, Just Plain Sites, Third party organizations, Information leakage, Facebook login

Permission to make digital or hard copies of part or all of this work for personal or classroom use is granted without fee provided that copies are not made or distributed for profit or commercial advantage and that copies bear this notice and the full citation on the first page. Copyrights for third-party components of this work must be honored. For all other uses, contact the Owner/Author(s). Copyright is held by the owner/author(s)

WPES'15, October 12, 2015, Denver, Colorado, USA.

ACM 978-1-4503-3820-2/15/10

DOI: http://dx.doi.org/10.1145/2808138.2808140.

\section{INTRODUCTION}

Whereas much attention has been paid to the risks posed by the web-based collection of private information by large organizations such as banks, large corporations, and the government (e.g., $[1,2,3])$, internet citizens also commonly visit web sites operated by small organizations such as momand-pop shops, blogs, sites for community activities, and school clubs and teams. We refer to this category of sites as "Just Plain Sites" (JPS's), after Lave's concept of "Just Plain Folks"[4]. Whereas large, well-funded organizations have the resources to operate in accordance with best privacy practices, JPSs are more likely to unintentionally expose their visitors' private information through inappropriate actions (e.g., collecting more private information than required), or inactions (e.g., failing to change default email templates to hide account passwords). ${ }^{1}$

In the present work we analyze JPS "front pages", in order to assess the prevalence of some of the privacy risks to which the operators of JPSs may be inadvertently exposing their visitors. These are the practices that apply at the very first encounter with a site, usually its home page (or, more generally, its landing page). Our "front page" principle accords with a "front of the store" metaphor. Visitors to a physical store should expect that in the front of the store, where they are simply browsing, they needn't be concerned with their credit card information being stolen (at least not by the store's proprietor), whereas once they head to the metaphorical "back of the store" to make a purchase or to participate in some other interaction with the management, they have made a conscious decision to hand over their credit cards, etc., and have, one hopes, assessed the safety of the situation, and decided with all appropriate concern and knowledge to put themselves at whatever risk they might feel necessary. Taking this concept back to the web: Once a visitor clicks "checkout" (etc.) they are, by assumption, aware of the privacy risks entailed by this transaction. Therefore, as we are investigating violations and issues before that point, we needn't go deeply into the web site, preferring instead to examine the privacy practices evident upon loading of the home or landing page.

Numerous studies have examined the privacy policies and practices of "specialist" web sites - i.e., sites whose subject-

\footnotetext{
${ }^{1}$ We will generally use the term "operator" to mean the owner, operator, developer, etc. of a site.
} 
matter is regulated, for example, government sites, sites that collect personal data from children under thirteen, and sites that collect health or financial data $[5,1,6]$. Other studies focus on the privacy practices of the most popular sites $[7,8$, 9]. We chose to examine "non-specialist" sites with relatively few visitors. Also, instead of studying the published privacy policies appearing on those sites, we investigated what these sites actually do (or not), not merely what they claim to do (or not).

The web is, of course, extremely complex, and things go on all the time that even experienced engineers are not aware of; almost certainly the owners, operators, and proprietors of JPSs are unaware of most of this under-the-hood activity. Becoming aware of this may encourage them to take steps to improve their practices, or at least to ensure that they are doing whatever they are doing with clear knowledge of the potential risks.

\subsection{Privacy Principles for JPSs}

Recognizing the importance of online privacy, many jurisdictions have moved to regulate the collection, storage, processing, and transfer of personal information [10, 11, 12]. Although the regulations in these jurisdictions differ in detail, they all address the topics of notice (for example, in the form of privacy policies), visitor choice in limiting the collection and transfer of their personal data, visitor access to their stored data, data security, and enforcement procedures [13, 14]. These regulations apply to both popular web sites, such as Google and Facebook, and to the less popular ones that we have termed JPSs. In its June 1998 Report to Congress, the Federal Trade Commission (FTC) reiterated five Fair Information Practice Principles (FIPPs): (1) Notice/Awareness; (2) Choice/Consent; (3) Access/Participation; (4) Integrity/Security; and (5) Enforcement/Redress [14], which were first stated by the U.S. Department of Health, Education and Welfare in 1973, and which have been influential in the formulations of the 1980 Organization for Economic Cooperation and Development (OECD) Privacy Guidelines and the Asia-Pacific Economic Cooperation (APEC) Privacy Framework of 2004 [12].

JPS operators should be aware of at least the FIPPs, OECD Guidelines, and APEC Framework, which we summarize in terms of five principles: 1. Minimize collection: The risk associated with communication and storage is proportional to the amount and type of data collected. Collect only the information needed for specific purposes, all of which should be made explicit in a privacy policy, or through other means, such as labeling at the point of collection. 2. Protect the data you collect: Sites that collect visitor data obviously should have reasonable data security practices with respect to storage, disposal, and break-ins. Indeed, the FTC requires such practices, however, enforcement is extremely difficult and so essentially non-existent [12], and is likely to be even more lax regarding JPSs, which present a huge number of tiny targets. Moreover, even among experts there is disagreement about what "reasonable" means [15]; the goal posts keep changing as criminal hackers up the ante, and even expert engineers with the best-of-intentions make mistakes. Faced with these complexities, some clear precautions include requiring strong passwords, encryption when transmitting and storing private data, avoiding the long-term retention of private data by disposing of it as soon as it has served its purpose, and developing a strong cyber- security plan. 3. Minimize both intentional and unintentional sharing: Third parties such as affiliates and service providers, are likely not held to the same privacy standards as a given site itself. Whereas it is less likely for JPSs to have formal affiliates, it is quite common for them to utilize service providers and third party services. For example, many small sites utilize Google Analytics, Facebook login, social icons such as "Like" buttons, and/or advertising frames without realizing that these may be turning over information about their visitors to third parties, most of which employ mysterious and complicated algorithms. This is often true even without the objects being clicked by the visitor (for example, on page load). Therefore, all uses of third party services that are not necessary for a site's core operations should be considered suspect. 4. Post a privacy policy that tells visitors what actually happens: To the extent possible, the published privacy policies should reflect what a site actually does, not merely what is intended or required. This is probably more difficult for JPSs operators who may not fully understand what their site software is doing. 5 . Give visitors choice and access: Visitors should be able to control their own data to the extent possible, for example, through opt-in and opt-out buttons [16], especially when a site intends to share data with a third party [17]. Visitors should also be able to check, change, or delete their private data, and their entire profile.

\section{EXPERIMENTS}

With these principles in mind, we conducted a number of experiments with the goal of describing the "front page" privacy practices of Just Plain Sites.

\subsection{Overview}

A small but critical aspect of a site's visitor privacy practices can be determined by technical analysis of the site itself on page load, including what sort of information is collected, or at least what sort of information is requested from the visitor. $^{2}$ Therefore, we set out to characterize the information collected by forms, pages, and policies presented in Just Plain Sites including the various trackers and analytics, cookies, and third party content employed by JPSs. In a second series of experiments we dug slightly deeper to examine JPS privacy practices related to the rapidly growing practice of "social login", especially regarding permissions and password storage practices.

We begin with some terminology that we will maintain throughout. We then develop a rough classification of JPSs, and enter into our central analysis, that of the use of third party services and cookies. Next we turn to first-party information explicitly collected via web forms, analyzing the form purpose and type of information collected, and ask whether the use of the requested information is sensible. Following that we examine the use of third party cookies. In the second half of the paper we change focus to login practices, and especially the increasingly common practice of Facebook login, examining the types of information requested by JPSs from Facebook, and characterizing the information requested by different types of JPSs. We also observe several sorts of bad

\footnotetext{
${ }^{2}$ Many less direct privacy-related things can also be determined on page load, for example, whether the site transmits information in encrypted form, although we did not analyze such factors.
} 
practices being employed by JPSs, including using deprecated methods, and passing passwords in the clear.

\subsection{Some Terminology}

We will usually refer to typical adult individual visitors to a web site as "visitors", or sometimes "users". The phrase "the site" will generally refer to the site that given visitor is explicitly aware that they have visited. If the site communicates with other sites (or web services of any sort that are not under control of the site operator), we will refer to these other sites/services as "third parties". Importantly, from the point of view of the visitor to the site, there is usually no apparent distinction between content provided by the site itself, and that provided by third parties. Here, in large part, lies a significant source of unintended privacy risk, because visitors usually can not tell when they are sending data to the site operators they intended to be communicating with, or some other, unintended, organization. Note that this is exactly the same situation as in criminal internet piracy there is an unintended third party "listening in" to at least some of the conversation (although in the present case no crime is usually taking place).

\subsection{Selecting Target Sites}

We wanted to obtain a list of U.S. sites in the "middle tier", not so small as to be trivial, but also not so large as to be likely to have resources that would enable them to easily hire privacy experts to manage their sites. The Quantcast top million list (www.quantcast.com; accessed on Jun 30, 2014) contains sites used in the U.S. with more than approximately 300 monthly visits. We removed sites with a ranking greater than 50,000, thereby excluding those having more than approximately 30,000 monthly visits. From those remaining we dropped "gov" sites, and those with "hidden profiles" (per Quantcast's terminology). This left 943,489 sites. Manual inspection of 100 sites sampled uniformly suggested that this process resulted in a selection of sites that roughly agreed with our sense of what a "Just Plain Site" should be. All of our experiments began with this list of nearly 1 million sites.

\subsection{Rough Classification of JPSs}

Because it is likely that different sorts of sites operate under different privacy regimens, it is useful to classify sites into categories that are likely to accord with such regimens. There are various ways to categorize web sites. Existing solutions mostly perform text/document categorization (e.g., similarweb), or utilize user-provided classes (e.g., dmoz). We found both of these to be too detailed for an analysis that covers such a large number of sites. We developed a classification based upon what product or service is being provided (content v. good), and who is providing it (the site operator v. visitors), as described in Table 1.

We manually classified the 100 uniformly sampled sites. The percentages in Table 1 represent the distribution of classes in this set. $13 \%$ were uninterpretable. Shopping-type sites dominate, but there are many blog-type sites as well. Small ad networks, like Craigslist or Ebay (but smaller) are rare. Surprisingly, social network- and forum-type sites are relatively uncommon.
Table 1: Categories of JPSs and Representation

\begin{tabular}{|c||l|l|}
\hline & $\begin{array}{l}\text { Content, } \\
\text { Web Service }\end{array}$ & $\begin{array}{c}\text { Goods, } \\
\text { Physical Service }\end{array}$ \\
\hline \hline $\begin{array}{c}\text { Single } \\
\text { Producer }\end{array}$ & $\begin{array}{l}40 \% \text { - Blog, } \\
\text { official site, } \\
\text { web tool, an- } \\
\text { nouncements } \\
\text { for a club, news }\end{array}$ & $\begin{array}{l}44 \% \text { - Online } \\
\text { shop, poster } \\
\text { site a shop, } \\
\text { paid online } \\
\text { game }\end{array}$ \\
\hline $\begin{array}{c}\text { User } \\
\text { produced }\end{array}$ & $\begin{array}{l}3 \% \text { - Social net- } \\
\text { work, forum, } \\
\text { wiki, group } \\
\text { discussions }\end{array}$ & $\begin{array}{l}\text { Ad networks, } \\
\text { consumer consumer } \\
\text { to clatforms }\end{array}$ \\
\hline \hline
\end{tabular}

\subsection{Third Party Service Analysis}

Sites often interact with third parties for various purposes, such as requesting static resources (e.g., code libraries, images, css, fonts), analytics, ads, social widgets, web beacons, and so on. It is generally difficult or impossible for the site to determine the extent of a third party's privacy practices. When someone visits a page that accesses third party services, some information (e.g., IP address, browser type - socalled "fingerprinting" [18])) is sent to the third party service provider, often without any action required by the visitor. Usually third parties use this information for personalized ads, improving their services, or aggregate them with other information. Many web pages contain "social" buttons to "like", "tweet", "digg", etc., and such trackers often add third party cookies when you visit the page, again without any action required by the visitor. Moreover, some of these third parties are better "ninjas" than others, not leaving tracks such as cookies, but nonetheless gaining access to the visitor's information. For example, Facebook states that: "If you've previously received a cookie from Facebook because you either have an account or have visited facebook.com, your browser sends us information about this cookie when you visit a site with the "Like" button or another social plugin." (emphasis added) Note that the specific way that third parties use cookies will vary. The Facebook Like button, for example does not install a cookie on load, it just checks for an existing cookie from previous visits to the Facebook site itself. The Twitter Tweet button, by contrast, installs a cookie on the visitor's browser.

We define a "tracker" as any process that the site, or a third party, may use that would create an unintentional privacy risk, for example, identifying the visitor directly via a browser profile. ${ }^{3}$ However, many commonly employed mechanisms pose an unintentional privacy risk. For example, unless the site operators goes out of their way to change the default behavior of the Apache server, it will create unencrypted $\log$ files of all access to the site, left in a commonly known default location. Static "Side-loading" of files (scripts, images, etc.) from third parties is a very common practice and creates an unintentional privacy risk by virtue of the visitor unknowingly accessing these third party sites, thereby permitting the third party to profile the visitor who is unwittingly accessing their site for this side-loading. Another very common example of this sort of tracking is the

\footnotetext{
${ }^{3}$ This definition excludes primary usage tracking, as that would be intentional.
} 
Table 2: Third party accesses

\begin{tabular}{|c|c|}
\hline Description & Count \\
\hline \hline Requests & 252036 \\
\hline Responses & 251802 \\
\hline URLs accessed & 503838 \\
\hline Distinct URLs & 201628 \\
\hline Distinct hosts & 9580 \\
\hline Distinct hosts (combined) & 8601 \\
\hline \hline
\end{tabular}

use of analytic sites, such as Google Analytics. Google, of course, utilizes this data for their own unknowable purposes, as well as for the intended purpose of the originating site. More insidious are "beacons", pieces of code that activate when the web page is loaded, regardless of the visitor clicking on them. These are often essentially invisible - tiny black images or space characters (so-called "pixel tags") and may be linked to arbitrary URLs or complex javascript code, enabling data-gathering computations without visitor awareness.

\subsection{Data Capture and Cleaning}

As mentioned in the introduction, we are concerned primarily with privacy practices that affect a visitor by just loading the site's landing page, and by clicking the available buttons. In accordance with this policy, we do not analyze referred domains unless there is an immediate redirect, and then we only follow one such redirect. Also, we only consider what is collected while the visitor is browsing, that is, we do not fill in info required to take whatever "next steps" might be possible from a page. In fact, we never fill in any information at all, but merely look at what happens upon clicking the available buttons.

We used PhantomJS to capture all http requests and responses on page load, ignoring "local" requests/responses (i.e., those within the same domain or a subdomain). We parsed the URLs into the domain, path, filename, and other information, and then combined URLs that appeared to be served by the same entity (for example, s1.criteo.com and s2.criteo.com, or where the IP addresses are the same except for the last octet).

\subsection{General Description of the Dataset}

Table 2 and Figure 1 provide a general sense of our data. Among the 8,601 URLs accessed, 8,451 were analyzable ${ }^{4}$. Among these, $82 \%$ had at least one third party access on page load. The number of third party requests from a single JPS page load ranges from zero to more than 150 (Figure 1). When someone visits a site, an average of 7 other organizations may know that they have been there.

\subsection{Monopoly in Third Party Services}

By combining the results from different sources, a third party organization can obtain significant additional information about a web site's visitors. For example, if a third party provider can trace a unique visitor across different sites by aggregating the data it is given "on the side" (e.g., via browser profiles), it can better estimate a visitor's interests

\footnotetext{
${ }^{4}$ The remainder failed for various complex, uninteresting, and/or inexplicable reasons.
}

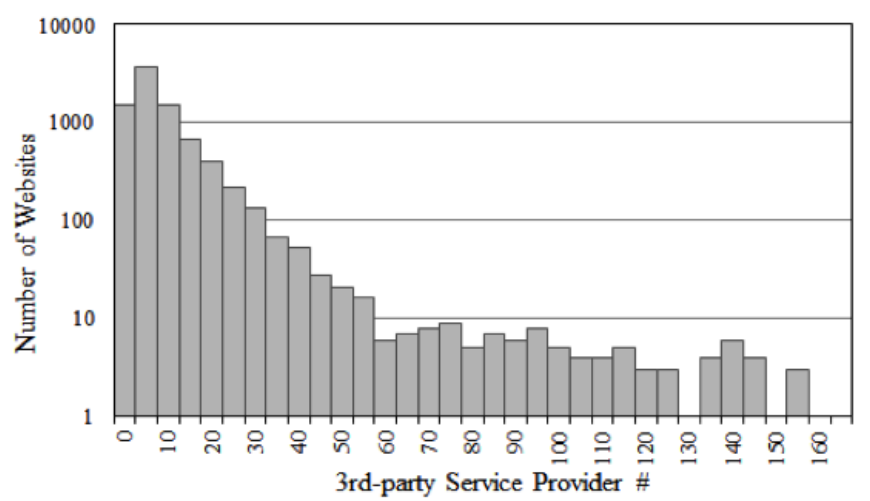

Figure 1: Third party accesses per service provider

and can improve targeted advertising. Therefore, we sought to identify the owner of third party services in our dataset.

To accomplish this we categorized URLs based on their hostname, after truncating subdomains. Since third party service providers often use several different domains, we had to aggregate the domains by each owner. This task is not straightforward, and we could not develop a fully automatic method. Therefore we manually aggregated the domains, considering issues such as mergers and acquisitions, and "dereferencing" Content Delivery Networks (CDNs). For example, Google uses numerous fronts, such as doubleclick.net, gstatic.com, ytimg.com, and blogger.com. And fbcdn. net, as well as any subdomain of akamaihd.net that contains "fbcdn" are fronts for Facebook.

Unsurprisingly, more than $67 \%$ of the JPS web sites use at least one service of Google. Another 19\% use at least one service of Facebook, and about 11\% use services from Twitter. Approximately $35 \%$ is divided among various smaller players, like Amazon, Quantcast, and Wordpress at about $4 \%$. (This may sum to more than $100 \%$ because sites may use third party services from multiple providers.)

\subsection{The Purposes of Third Party Requests}

Some kinds of third party requests present more of a privacy risky than others. For example, side-loading "show_ads.js", being a script, is almost certainly more dangerous than a style sheet or image fetch [19]. We sought to characterize in the purpose of third party requests to the top two players: Google and Facebook. We considered combinations of several criteria such as domain (and subdomains), path/filename, and URL parameters. It is usually not possible to correctly recognize the purpose of a third party request just from the domain or filename. For example, Google Analytics uses "-utm.gif". The content of this tiny image is merely a "beacon", a great deal of information, often comprising hundreds of bytes, is passed in the request URL.

We first recognized static resources and then worked on ads, analytics, and other third party requests, as their recognition is more challenging. Static resource URLs usually have no parameters, or have only short, simple parameters, and usually do not set cookies in their headers. The static categories that we found included static CSS (.css), images (.jpeg, .jpg, .png, .gif), javascript (.js), json, (.json), and html (.html). 
In addition to the above static URLs, we observed these (apparent) functions ${ }^{5}$ : $A d s$ : if the domain is for an advertising company and there are related keywords in the subdomain (e.g. ads.yahoo.com) or in the path (e.g. doubleclick. net/pagead/ads); Analytics: if the domain is for an analytics company and there are related keywords in the subdomain (e.g. analytics.bigcommerce.com) or in the path (e.g. .../track); Beacons: if there are keywords in the subdomain (e.g. pixel.quantserve.com) or in the path (e.g. .../bug/pic.gif) and the URL parameters are not empty; and Widgets: if there are keywords in the subdomain (e.g. widgets.wp.com) or in the path (e.g. .../js/plusone.js).

Figure 2 depicts the distribution of usage patterns of third party requests.

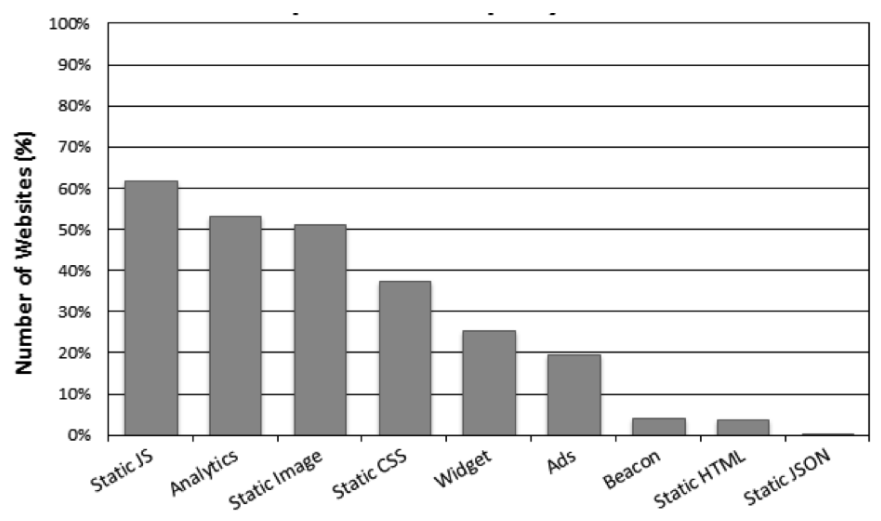

Figure 2: Purpose of third party requests

Figure 3 depicts the usage pattern of Google and Facebook, the two biggest third party service providers. The most popular Google service is analytics. In terms of providing widgets, the popularity of Google and Facebook is almost the same.

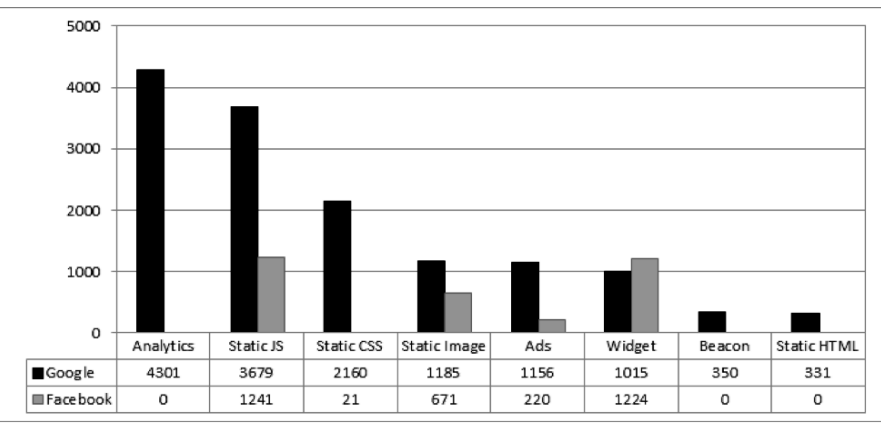

Figure 3: Usage pattern of Google v. Facebook services

\section{FIRST-PARTY FORMS}

So far we have been discussing only implicitly-collected information, but much more invasive information, such as so-called "Personally Identifiable Information" (PII), is often explicitly requested via html forms on the landing page.

\footnotetext{
${ }^{5}$ As only the author really knows what the javascript really does, we characterize only what it appears to do.
}

In order for a visitor to decide whether the personal information being requested is necessary, it is necessary to know the purpose to which the information will be put. For example, it should be a red flag for a site to request your physical mailing address in order to subscribe to an electronic newsletter. There may be a rational reason for this, but it would be useful to have an explanation before making the choice about whether to reveal this sort of information.

\subsection{Web Form Purpose and Information Type}

According to our analysis, $54 \%$ of JPSs collect visitors' PII through HTML forms. We tried to classify the purpose of web forms and the information type of their fields. In order to classify the information type of the form (Figure 4) we considered the name, label, and default value of the fields. When a field had no label we considered the text of a previous sibling if a label was available there. Finally, if we still could not classify the information requested, we checked the first parent of the DOM element and also the text in the field, if any. In order to classify the purpose of the form (Figure ), we considered the text of the first child of the form (hopefully a title or short description of the form's purpose), the previous sibling of the form (often a title for the form is there), and also the text body surrounding the form, if it was not unreasonably long. Table 3 depicts the type-by-form results.

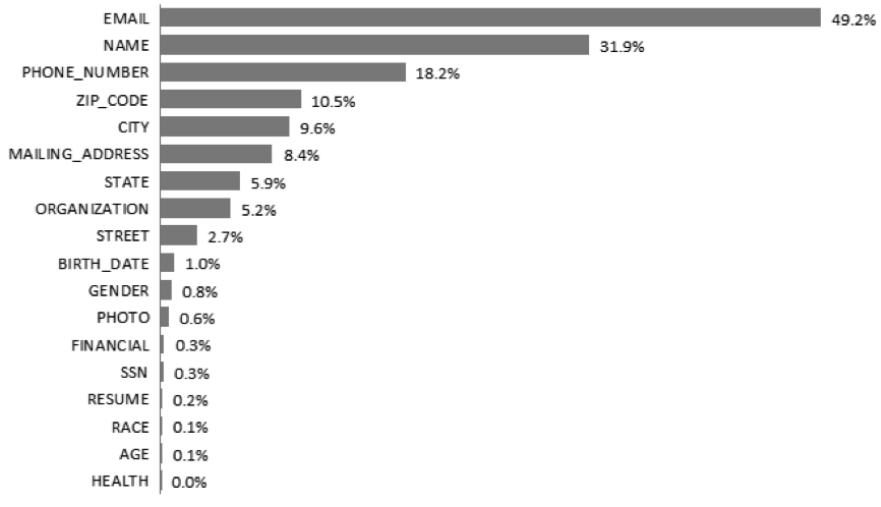

Figure 4: Information collected in web forms

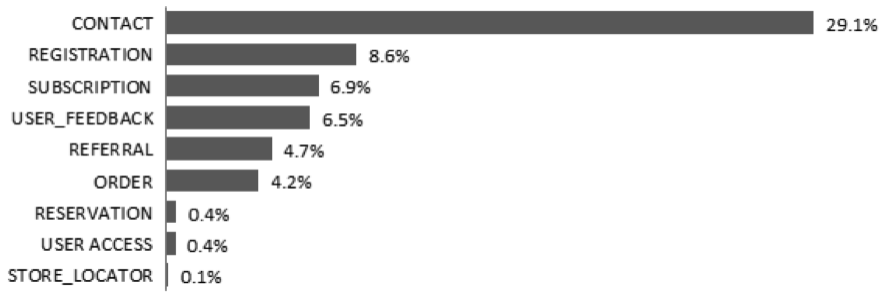

\section{Figure 5: Purpose of collected information}

In order to assess the quality of our method, we manually coded a sample of 100 forms. In fifty-eight (58\%) of these, our automatic and manual analyses matched exactly. Among the rest, there were 131 errors, both false positive and false negative, totaling approximately $6.24 \%$. 


\subsection{Uses of Requested Information}

Unsurprisingly, visitor names and email addresses are the most commonly requested information. These are used for many purposes such as registration, feeds, etc. After these, the most-collected PII is phone numbers, and a smaller number of sites collect other PII, such as birthdates.

We wanted to sample the reasons for birthdate being collected, and to assess whether it seemed to make sense to collect this information, given the purpose of the site. Table 4 indicates the results of this exploration. In most cases where birthdate was collected we could determine whether it was collected in full or partially, and whether the purpose for collecting such information seemed to make sense. For example, it clearly makes sense for an insurance company to want to know an applicant's birthdate. Similarly, it makes sense for a "men's supper club", to want to know a partial birthdate, which would indicate the registrant's age, although not his or her exact birthday. These are both sensible uses of information. On the other hand, it makes less sense for a summer camp to require full birthdate information; it may need to know the applicant's age, but then it could request partial birthdate information, or equivalently, simply request the applicant's age. Table 4 also indicates whether the site explicitly indicated the reason for collecting this data. Notice that in almost no case was this explicitly stated, although in a few we deemed it obvious.

\section{THIRD PARTY COOKIES}

As mentioned above, a specific sort of tracking is represented by third party cookies, which many sites leave on visitors' browsers. We conducted a survey of third party cookies based upon two uniform random samples of 1000 and 10000 JPS from our original dataset. As in the previous experiments, we used PhantomJs to capture external resources fetched during webpage load, including Javascript libraries, images, css files, trackers, and ad network referrals. We evaluated how many web sites use third party cookies (around 50\%), the lifetime of third party cookies in comparison to "own cookies" (those from the visited site), and the popularity of various third party cookie domains. Unless otherwise stated, the following results arise from the $10 \mathrm{k}$ dataset.

Those domains providing more than $5 \%$ of third party cookies were (rounded): .doubleclick.net: $18 \%$, google. com: $9 \%$, .twitter.com: 7\%, .score-card-research.com: $7 \%$, adnxs.com: $7 \%$, and .youtube.com: $6 \%$. One can see that most third party cookies come from ad or social networks. The lifetimes of cookies were not very variable: most cookies' lifetimes are over a year (own: $52 \%$ v. 3rd: $33 \%)$. Next to this, most own cookies have "session" durations (own: $8 \%$ v. 3rd: $2 \%$ ), whereas most third party cookies have one hour durations (own: 1\% v. 3rd: 6\%), followed by 1 year durations (own: $2 \%$ v. 3 rd: $5 \%$ ).

\section{THIRD PARTY LOGINS}

Third party login, such as "signup/login with Facebook", is becoming increasingly popular among JPSs. Most visitors are presumably aware that some information is shared between the local site and Facebook when such a login is employed, at least when specific permissions are asked in a dialog box. However, visitors are probably not aware of the details of this sharing. ${ }^{6}$

Detecting Facebook login on a site is complicated by the variety of ways in which it can be implemented. However, it is easier to detect automatically in comparison with, for example, OpenID with a variety of interfaces, or Janrain with Ajax implementations, because the code for Facebook login is highly determined by Facebook, so we can detect it by checking for a specific URL in a dialog window, or in the page after clicking login links or buttons on the web site.

For this analysis we uniformly selected a subset of 100,000 (100k) sites from our original dataset. In accordance with our assumptions about the simplicity of JPS sites, we assumed that a login/registration page would be accessed by at most one click from the landing page. Web Site operators wishing to permit Facebook login may either use the Facebook-provided html, or may code their own custom implementation. We used PhantomJSDriver and FirefoxDriver to render the DOM and click on various controls and tags. We targeted XPath: "//input[ @type= 'submit'] | //a | //button" - for custom implementations and "//iframe[ @title= 'fb:login_button Facebook Social Plugin'] |//div[ @class= 'fb-login-button']" - for Facebook login buttons, and considered only tags with login-related text such as: "signup", "connect with", "facebook", etc. Although this method does not take into account complex Ajax interactions, state information is still saved by the target URLs. We used Selenium to click even on hidden Facebook login buttons, if they were present in the page source. This covers situations where the DOM is changed, for example revealing a hidden div that appears on clicking "signup".

We ended up with two sets of sites that use Facebook login: 1,191 sites obtained from the BuiltWith statistics, and 260 additional sites that use a custom Facebook login implementation, found in our 100k sample. From these two sets $(1,191 \& 260)$ we asked (a) what specific information is requested by Facebook, and (b) how many sites still generate and explicitly store passwords, in comparison to more secure means such as sending an activation link? ${ }^{7}$ The second question was answered by analyzing "congratulating new user" emails received after login, where we also noticed the use of several problematic practices. For all of the above we manually checked 20 random web sites from each sample.

\subsection{Information Requested from Facebook}

Detailed results are provided in Table 5, giving the top 10 specific permissions are requests. Naturally, specific permissions are requested more often in the case of a custom implementation of Facebook login: only $8 \%$ web sites use default "public profile" permissions, whereas $31 \%$ of "ready to copy-paste" Facebook login buttons use the defaults. More interesting are the exact permissions JPSs ask for, which are in some situations, redundant. For example, "user_birthday" is very popular, despite the Facebook's guidelines: "Use any available public profile information before asking for a permission. For example, there is an age_range field included

\footnotetext{
${ }^{6}$ Several studies, e.g., [20], have studied the permissions requested by Facebook apps, but not local web sites that use Facebook login.

${ }^{7}$ Social login can also be done via third party platforms, such as eventbrite.com. We did not restrict redirections for signup/login.
} 
Table 3: Distribution of PII types based on form type

\begin{tabular}{|c||c|c|c|c|c|c|c|c|c|c|}
\hline & EMail & Name & Phone & Zip & City & Addr. & St. & Org. & St. & Bd. \\
\hline \hline Contact & $96 \%$ & $53 \%$ & $31 \%$ & $17 \%$ & $16 \%$ & $16 \%$ & $11 \%$ & $8 \%$ & $5 \%$ & $1 \%$ \\
\hline Order & $83 \%$ & $43 \%$ & $39 \%$ & $19 \%$ & $14 \%$ & $9 \%$ & $7 \%$ & $14 \%$ & $5 \%$ & $3 \%$ \\
\hline Referral & $84 \%$ & $50 \%$ & $12 \%$ & $13 \%$ & $7 \%$ & $4 \%$ & $3 \%$ & $4 \%$ & $2 \%$ & $1 \%$ \\
\hline Registration & $43 \%$ & $12 \%$ & $6 \%$ & $3 \%$ & $3 \%$ & $2 \%$ & $1 \%$ & $2 \%$ & $0 \%$ & $0 \%$ \\
\hline Reservation & $79 \%$ & $45 \%$ & $55 \%$ & $18 \%$ & $13 \%$ & $13 \%$ & $8 \%$ & $3 \%$ & $0 \%$ & $0 \%$ \\
\hline Store Locator & $11 \%$ & $0 \%$ & $11 \%$ & $56 \%$ & $22 \%$ & $22 \%$ & $11 \%$ & $0 \%$ & $11 \%$ & $0 \%$ \\
\hline Subscription & $95 \%$ & $31 \%$ & $7 \%$ & $6 \%$ & $4 \%$ & $4 \%$ & $2 \%$ & $2 \%$ & $1 \%$ & $0 \%$ \\
\hline Unknown & $79 \%$ & $48 \%$ & $26 \%$ & $16 \%$ & $14 \%$ & $10 \%$ & $7 \%$ & $7 \%$ & $4 \%$ & $2 \%$ \\
\hline User Access & $34 \%$ & $17 \%$ & $11 \%$ & $14 \%$ & $9 \%$ & $6 \%$ & $6 \%$ & $6 \%$ & $6 \%$ & $3 \%$ \\
\hline User Feedback & $94 \%$ & $69 \%$ & $32 \%$ & $4 \%$ & $4 \%$ & $3 \%$ & $2 \%$ & $5 \%$ & $1 \%$ & $0 \%$ \\
\hline \hline
\end{tabular}

Table 4: Manually examined examples of birthdate requests

\begin{tabular}{|c|c|c|c|c|}
\hline Page Purpose & Full BD? & Purpose & Rational? & Explained? \\
\hline Life Insurance Quote & Full & Need for Process & Yes & No but Obvious \\
\hline $\begin{array}{ll}\text { Heath } & \text { Insurance } \\
\text { Quote } & \end{array}$ & Full & Need for Process & Yes & No but Obvious \\
\hline $\begin{array}{ll}\text { Auto } & \text { Insurance } \\
\text { Quote } & \end{array}$ & Full & Need for Process & Yes & No \\
\hline $\begin{array}{l}\text { International Stu- } \\
\text { dent Request Info }\end{array}$ & Full & Identity & Yes & No \\
\hline $\begin{array}{l}\text { Loan Information } \\
\text { Request }\end{array}$ & Full & - & No & No \\
\hline Subscription & Partial & Special Offers & Yes & No \\
\hline $\begin{array}{l}\text { Special Offer Sub- } \\
\text { scription }\end{array}$ & Full & Check Age & Yes & Yes \\
\hline Join Club & Full & Check Age & Yes & Yes \\
\hline $\begin{array}{l}\text { Alumni Information } \\
\text { Update }\end{array}$ & Full & $\begin{array}{l}\text { DoubleCheck } \\
\text { Identity }\end{array}$ & $?$ & No \\
\hline $\begin{array}{l}\text { Apply for Graduate } \\
\text { Program }\end{array}$ & Full & Identity & Yes & No \\
\hline $\begin{array}{ll}\text { Subscription } & \text { Infor- } \\
\text { mation Card } & \end{array}$ & Full & - & No & No but Obvious \\
\hline Camp Registration & Full & - & $\begin{array}{l}\text { No Just } \\
\text { needs age }\end{array}$ & Yes \\
\hline $\begin{array}{l}\text { Special Offer Sub- } \\
\text { scription }\end{array}$ & Partial & Special Offers & Yes & No \\
\hline $\begin{array}{l}\text { Funeral Arrange- } \\
\text { ment }\end{array}$ & Full & - & Yes & Yes \\
\hline Membership & Full & - & No & No \\
\hline Membership & & Need for Process & Yes & No \\
\hline Subscription & Full & Need for Process & Yes & No \\
\hline For Magical Spells & Full & $\begin{array}{l}\text { Need for Magical } \\
\text { Process }\end{array}$ & Yes & No \\
\hline $\begin{array}{l}\text { Free Spell Consulta- } \\
\text { tion Form }\end{array}$ & Full & Need for Process & Yes & No \\
\hline Application Form & Full & - & No & No \\
\hline
\end{tabular}


Table 5: Information requests at Facebook login (Left over 260 sites, Right over 1,191 sites)

\begin{tabular}{|r|r|}
\hline \multicolumn{2}{|c|}{ Custom Implementation } \\
\hline Permission & Percentage \\
\hline email & $90 \%$ \\
user_birthday & $28 \%$ \\
publish_stream & $23 \%$ \\
user_location & $17 \%$ \\
read_stream & $10 \%$ \\
user_about_me & $9 \%$ \\
(offline_access) & $9 \%$ \\
user_likes & $9 \%$ \\
<default> & $8 \%$ \\
publish_actions & $8 \%$ \\
\hline
\end{tabular}

in the public profile..." Also, some JPSs are still using deprecated permissions, such as "offline_access".

\subsection{Clustering for Facebook JPSs}

As expected, Facebook login permission requests differ for different categories of web site, and at the same time result in natural clusters. According to our four-way categorization, depicted in Figure 1, among the 260 sites in the smaller sample, $48 \%$ were shopping sites, $34 \%$ were blogs, $17 \%$ were social, and $1 \%$ were ad sites. We decided to explore organic clustering based upon the permissions requested by these sites, as described above. We began with the 48 sites requesting Facebook permissions from those requested by JPSs with custom Facebook login code (from the experiment with $100 \mathrm{k}$ samples). We first combined the permissions requested by less than $5 \%$ of the sites into a single attribute "other". ${ }^{8}$ We clustered the resulting data using Weka's XMean algorithm. This resulted in a stable clustering based upon 13 features. The final clusters, ordered by amount of information requested, are summarized in Table 6 (next page).

Table 7 presents the distribution of site categories among each cluster. Several interesting observations can be made from this data. For example, just asking for email seems to be insufficient for most social networks; only $9 \%$ of the "User management" category (i.e., asking for email only, or nothing at all) are social networks. Also, the policy adopted by "Promotion" sites (requesting mainly email and the ability to publish on the visitor's stream, and sometimes other details) is equally popular among the four categories of JPSs (except ads which are rare in the data to begin with). As we proceed from top to bottom in the table, corresponding to more information being requested, blogs become less common, whereas social networks become more common. Shopping sites appear to be bi-modal between those that request minimum versus maximum information ( $48 \%$ v. $49 \%)$.

We also asked the opposite question: What permissions are popular in the different web site categories? Figure 6 represents the top 20 (of 48) differences (below this threshold the difference are too small to interpret).

Social sites usually request more top Facebook information than other categories, and defaults are not often requested by this type of site. Defaults and publish actions

\footnotetext{
${ }^{8}$ If the site requested only default permissions (actually, asking for a public profile), we did not consider "default" as a separate attribute.
}

\begin{tabular}{|r|r|}
\hline \multicolumn{2}{|c|}{ Login Button } \\
\hline Permission & Percentage \\
\hline email & $67 \%$ \\
user_birthday & $33 \%$ \\
<default> & $31 \%$ \\
publish_stream & $22 \%$ \\
(offline_access) & $13 \%$ \\
user_about_me & $12 \%$ \\
user_location & $10 \%$ \\
user_likes & $8 \%$ \\
read_stream & $7 \%$ \\
status_update & $5 \%$ \\
\hline
\end{tabular}

Table 7: Distribution of categories by cluster (\%)

\begin{tabular}{|c|c|c|c|c|}
\hline Cluster & Blog & Shop & Social & Ads \\
\hline \hline User management & 42 & 48 & 9 & 1 \\
\hline Promotion & 29 & 24 & 24 & 2 \\
\hline Profile management & 20 & 49 & 30 & 2 \\
\hline \hline
\end{tabular}

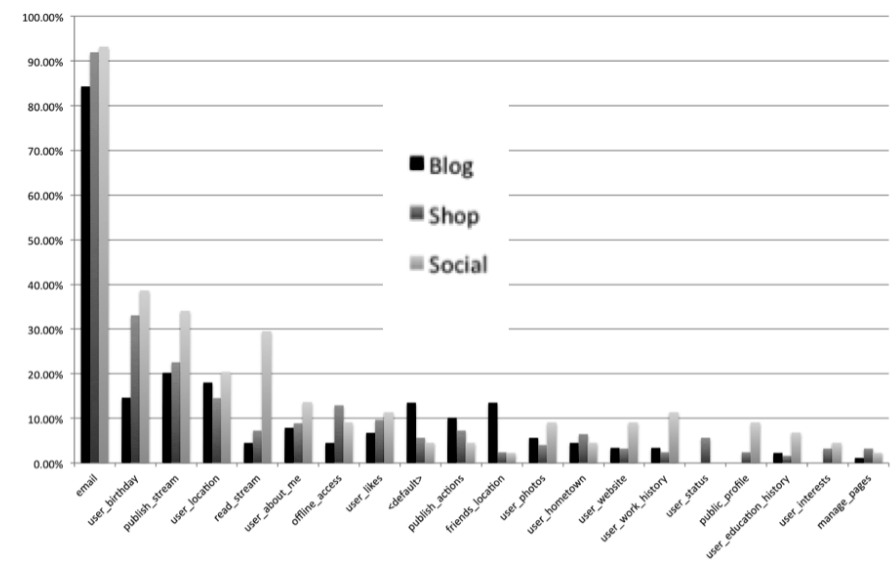

Figure 6: Differential usage of information in Facebook login requests

are commonly requested by blogs. Somewhat surprisingly, so is friend location. Offline access (which is deprecated), and visitor status, are often requested by shopping sites. Shopping sites actually vary the most for requested information. This may be a result of natural variability in the type of products offered.

It seemed odd to us that so many blogs were interested in friend locations, so we examined some of them manually, and noticed that many were political, for example: don-brown4tx14.org (Don Brown for congress), clark-republicans . org, citizen-actionwi.org, raise-your-vote.com, savepacifica.com, and yes-on-1tn.org. It makes some sense to ask for friends in order to build a political network. A finer-grained categorization might have put these sorts of sites into a separate category. 
Table 6: clusters ordered by amount of information requested

\begin{tabular}{|c|c|l|c|}
\hline Number & $\%$ & Requested Data & Pseudonym \\
\hline \hline 148 & $57 \%$ & Email only, or nothing & "User Management" \\
\hline 51 & $20 \%$ & $\begin{array}{l}\text { Mainly email and ability to publish on visitor's stream, and sometimes } \\
\text { other details. }\end{array}$ & "Promotion" \\
\hline 61 & $23 \%$ & $\begin{array}{l}\text { More profile information, such as email, birthdate, location, plus some- } \\
\text { times the ability to publish on the visitor's stream, and/or other details. }\end{array}$ & "Profile Management" \\
\hline \hline
\end{tabular}

\section{PASSING PASSWORDS IN THE CLEAR}

Actual registration is at the limit of complexity that we were able to automatically explore. While there are many "safe" ways to deal with passwords, such as sending re-passwording links, or sending one-time temporary passwords, many JPSs create a password for a new user, and then email it, unencrypted, to the user. Surprisingly, this is even the case for some sites that use social logins. Some popular platforms, such as Magento or Wordpress, provide tools for safe password exchange with email templates, but their defaults are unsafe, and JPS operators generally will not change these. We examined the prevalence of bad password practices by inspecting emails received after site registration: approximately $10 \%$ of sites using custom registration implementations sent a plain text password via email, whereas only $2 \%$ of those employed an activation link. Among sites using a login button, $4 \%$ sent a plain text password in email, versus $1 \%$ sending an activation link. Our statistics here are somewhat biased because we were unable to automatically answer a CAPTCHA test or enter a separate email during the process of registration. Regardless, these results provide a lower bound on the surprising number of sites that employ unsafe password practices.

\section{DISCUSSION}

\subsection{Result Highlights}

We have observed that JPS share a great deal of information with third parties, mostly without their visitors' knowledge, and probably without the JPS operators understanding what is being shared, nor the implications of this sharing. When you visit a site of the sort that we have studied, an average of 7 third party organizations find out about you, at least in terms of your browser profile. $82 \%$ of JPSs send at least one request to third party sites when they load. More than $67 \%$ of JPSs use at least one service of Google, and $19 \%$ use at least one service of Facebook. Setting aside static resource usage, which is generally considered less of a privacy concern, the most popular third party service is analytics, used by $53 \%$ of JPSs. Whenever social icons appear on a page, for example a Facebook "like" button, Facebook may be finding out that you have visited that page, even just on page load, without any button having to be clicked!

JPSs are collecting a great deal of information from their community. $49 \%$ of JPSs explicitly ask for visitors' email. When Facebook login is used, 23\% of JPSs request full profile management permissions from Facebook, and some are even requesting friend location information.

JPSs permit a great deal of tracking of their community. Third party cookies are used by more than $50 \%$ of JPSs, and the most popular third parties, Google and Twitter, use cookies for tracking. Both "own" and third party cookies usually live more than one year. Furthermore, around $30 \%$ of JPSs that use Facebook login ask for user_birthday, even given Facebook's guidance against this.

Deprecated permissions are still commonly requested, and JPSs often fail to update to the latest privacy practices. At least $5-10 \%$ of web sites with Facebook login still store and send passwords explicitly. This situation is worse with selfimplemented user management; the default Magento email template sends the password explicitly, and the same is true for some Wordpress plug-ins.

\subsection{Limitations and Improvements}

Automatic analysis of complex web sites is very difficult, largely because of the use of iframes, rest widgets, and javascript. Fortunately, our focus on JPSs ameliorates this problem slightly, as a smaller fraction of JPSs use sophisticated methods, or the widgetry they do use is well understood, such as third party login methods used in the default manner recommended by the provider. Ajax-based crawling, although much more complex, would enable a greater range of data gathering. It would also be useful to complement our observations with "ground truth", for example by way of surveys of the operators of JPSs.

\subsection{Conclusions}

Generally speaking our analyzes support our expectation that many of the practices of Just Plain Sites are potentially dangerous to visitor privacy. Our goal is not to scold JPS operators, but to raise awareness, both among JPS operators and among visitors to such sites. Both of these constituencies would probably be surprised, if not shocked, at what they may be inadvertently putting at risk. For JPSs on the web, collecting private data may sometimes be important to the site operators, and sometimes they may be aware of it - we are not claiming that visiting these sites always constitutes an actual danger of invasion of privacy; it may well be that such information leakage offers a benefit to the site operators and visitors, for example, by improving targeted advertising. But much of the time it is probably not important, and the site operators are not aware of it. Compare this with top tier sites where, even if the visitors are not aware or concerned with what is being taken from them, it is nearly certain that the site operators are well aware of these details. Indeed, in many cases this is their explicit business model, and when it is not their business model to sell out their visitors, such top tier sites have the resources to ensure that they understand and properly implement best privacy practices. This is not so for JPSs. Hopefully our work can move JPS visitors a small way towards having a richer understanding of what is going on when they visit these sites, and at the same time move JPS operators a small way towards awareness of potential problems, and towards properly implementing best privacy practices. 


\section{ACKNOWLEDGMENTS}

This work was supported by CommerceNet. Several anonymous reviewers helped us improve the presentation.

\section{REFERENCES}

[1] L. F. Cranor et al., "Are they actually any different? comparing thousands of financial institutions' privacy practices," The Twelfth Workshop on the Economics of Information Security WEIS 2013 Washington, DC, pp. 11-12, June 2013.

[2] A. L. Young et al., "Information revelation and internet privacy concerns on social network sites: A case study of facebook," in Fourth international conference on Communities and technologies, pp. 265-274, ACM, 2009.

[3] L. Humphreys, P. Gill, and B. Krishnamurthy, "How much is too much? privacy issues on twitter," in Conference of International Communication Association, (Singapore), 2010.

[4] J. Lave, Cognition in practice. Cambridge University Press, 1988

[5] A. I. Anton, J. B. Earp, and A. Reese, "Analyzing website privacy requirements using a privacy goal taxonomy," (IEEE Joint International Conference on 2002), pp. 23-31, 2002.

[6] J. Turow and A. P. P. Center, Privacy policies on children's Websites: Do they play by the rules? Annenberg Public Policy Center of the University of Pennsylvania, 2001.

[7] B. Krishnamurthy and C. E. Wills, "Generating a privacy footprint on the internet," in 2006 Internet Measurement Conference, IMC'06, 2006.

[8] D. Malandrino et al., "Privacy awareness about information leakage: Who knows what about me?," in WPES '13 Proceedings of the 12th ACM workshop on Workshop on privacy in the electronic society, pp. 279-284, 2013.

[9] B. Krishnamurthy, K. Naryshkin, and C. Wills, "Privacy leakage vs. protection measures: The growing disconnect," in Web 2.0 Security and Privacy Workshop, 2011.

[10] D. E. N. 95/46, "on the protection of individuals with regard to the processing of personal data and on the free movement of such data," vol. OJ, October 1995.

[11] M. Tokson, "Automation and the fourth amendment," Iowa L. Rev., vol. 96, p. 581, 2010.

[12] D. J. Solove and W. Hartzog, "The FTC and the new common law of privacy," Colum. L. Rev., vol. 114, pp. 583-1033, 2014.

[13] U. D. of Commerce, The U.S.-EU Safe Harbor Guide to Self-Certification. 2009.

[14] M. K. Landesberg, T. M. Levin, C. G. Curtin, and O. Lev, "Privacy online: A report to congress," Federal Trade Commission, 1998.

[15] M. D. Scott, "The FTC, the unfairness doctrine, and data security breach litigation," Admin. L. Rev., vol. 60, pp. 127-182, 2008.

[16] G. R. Milne and A. J. Rohm, "Consumer privacy and name removal across direct marketing channels," Journal of Public Policy and Marketing, pp. 238-249, 19.22000 .
[17] J. R. Mayer and J. C. Mitchell, "Third-party web tracking: Policy and technology," in IEEE Symposium on Security and Privacy, 2012.

[18] N. Nikiforakis et al., "Cookieless monster: Exploring the ecosystem of web-based device fingerprinting," in Symposium on Security and Privacy, IEEE, 2013.

[19] N. Nikiforakis et al., "You are what you include: Large-scale evaluation of remote javascript inclusions," in 19th ACM Conference on Computer and Communications Security, CCS'12, 2012.

[20] M. Huber et al., "Appinspect: Large-scale evaluation of social networking apps," in ACM Conference on Online Social Networks: COSN'13, 2013.

\section{APPENDIX: JUST PLAIN (MOBILE) APPS}

JPSs are often accessed via smartphone or tablet apps. Indeed, in some cases this is the primary means of access. Therefore, it would make sense to analyze mobile clients. Unfortunately, the app world is so different from the web that we were unable to make our methods apply without basically starting over. First off, scraping apps, as compared to scraping web sites, is complicated, even in the case of Android's xml-based layout of screens. Because of greater interface design freedom, it is hard to properly detect mobile analogues of forms, and the correspondences among positionally separated labels and inputs. Moreover many app developers us dynamic text. On the other hand, app user management, third party logins, analytics, and other practices are similar to web sites.

We tried to answer the questions of how many JPS web sites have separate mobile versions, and how many JPSs have supporting mobile apps. However, only about $10 \%$ of the JPS sites that we accessed had mobile redirection (e.g., "/m.", ".mobi"). Many sites now use "universal layout", with separate divs, or an entirely separate site for mobile access. We are unable to see such solutions in our existing dataset. In a second analysis we searched Google Play for apps matching each JPS web site by name. The percentage was very low as well, perhaps because in many cases, as mentioned, the developers of the app for a JPS are not the same as the JPS. These preliminary analyses yielded low rates and difficult-to-interpret results.

Fortunately for the user, mobile developers have to try somewhat harder to get data off the phone or pad than than when a small mom-and-pop shop puts up their own web site, so mobile developers are somewhat more likely to know what they are doing in this regard, or at least know that they are doing it. Also, apps that are distributed by online stores, such as iTunes and Google Play, are subject to fairly strict policies regarding data collection and privacy.

For all these reasons we put our analysis of JPS apps (JPAs?) on hold for another time. 